\title{
DETECTOR FOR VERY SMALL ALTERNATING CURRENTS AND ELECTRICAL WAVES.
}

By L. W. Austin.

A number of years ago I noticed that a copper sulphate cell with copper electrodes, one of which was considerably larger than the other, showed an apparent rectifying effect for alternating currents of the order of those produced by speaking with the mouth pressed against an ordinary telephone receiver. In the original experiment the rectifying cell, the telephone, and a d'Arsonval galvanometer of moderate sensibility were connected in series. The lowest tone spoken into the telephone produced a deflection of the galvanometer, and a louder one drove it from the scale. Recently I have again taken the matter up, desiring, if possible, to find an explanation of the rectifying effect, and also to test the usefulness of the detector in the laboratory.

To test the sensibility of the detector for alternating currents, a known alternating current was passed through a bridge wire of about $1 \mathrm{ohm}$ resistance, and from this, by means of sliding contacts, various potential differences were applied to the detector (see fig. 1). A form of key was used which kept the

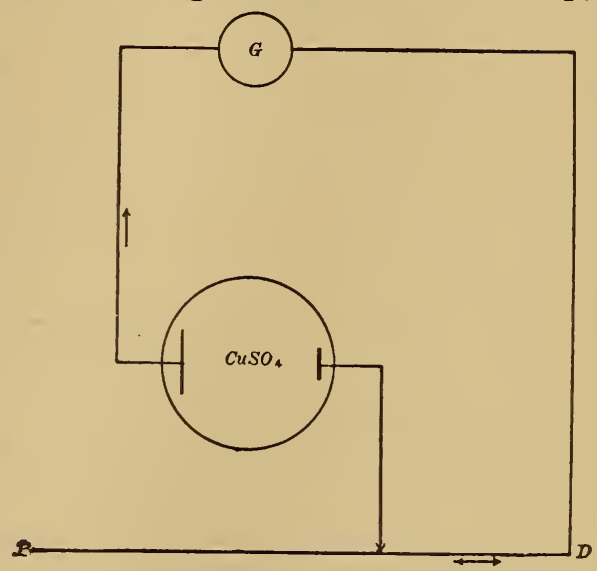

Fre. 1.-Connections for detector (form 1). detector circuit short-circuited when not connected to the potential wire. This was necessary on account of the small electromotive forces always present in the cell, due to slight differences in the electrodes. In order to reduce these as much as possible, the electrodes were generally made from the same No. 30 wire, the small one varying from $1 \mathrm{~mm}$ to 3 or $4 \mathrm{~cm}$ in length, and the larger one from 10 to $30 \mathrm{~cm}$. 
The sensibility did not seem to vary in any marked degree with the size of the small electrode within the limits indicated.

The first hy pothesis entertained regarding the nature of the phenomenon was that it was a true rectification, the resistance being greater for a current entering the small electrode than for one leaving it. But the following experiment seems to indicate that the direct current is due, in part at least, to some chemical action produced by the alternating current and analagous, perhaps, to the solution of $\mathrm{Pt}$ in $\mathrm{H}_{2} \mathrm{SO}_{4}$ noticed by Margules ${ }^{a}$ and $\operatorname{Ruer}^{b}$, the action being greater on the small electrode on account of the greater current density. It seems probable, however, that the cause of the phenomenon may contain more than one factor. This is indicated by the fact that a reversal of

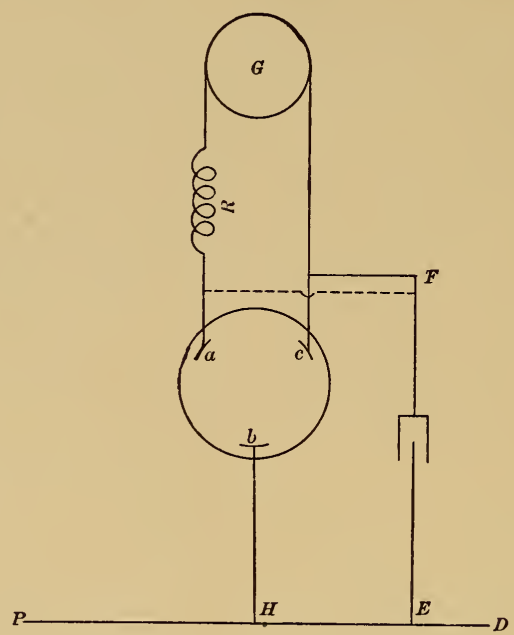

FIG. 2.-Connections for detector (form 2).

the usual direction of the current is occasionally observed, especially with fine point electrodes and high frequencies. A cell was constructed with three electrodes, $a, b, c$ (see fig. 2), $b$ and $F$ being connected to the potential wire $P D$ with a condenser inserted in $E F$ and a high inductive resistance in the galvanometer circuit, the arrangement being such that most of the alternating current could be sent through either one of the electrodes of the galvanometer circuit, and very little through the other. It was found that either $a$ or $c$ could be made electropositive by connecting $F$ to it, and that the direction of the current was now independent of the relative size of the electrodes, at least within wide limits.

$a$ M. Margules: Wied. Ann. 65, p. 629, 1898; 66, p. 540, 1898.

$b$ M. Ruer: ZS. f. phys. Chem. 44, p. 81; 1903. 
In the accompanying curve (fig. 3) the relation is shown between the direct current produced and the alternating potential difference applied to a two-electrode cell (fig. 1), connected in series with a galvanometer having a sensibility of about $1.10^{-8} \mathrm{amp}$. Above 0.015 volt (not shown in figure) the curve is nearly a straight line until in the neighborhood of 0.3 volt it bends suddenly and becomes nearly parallel to the horizontal axis. The lowest voltage observable under favorable circumstances with the instrument connected directly (i. e. without a transformer) and with a sensitive galvanometer is about 0.0001 volt, the sensibility being about the same as that of a good telephone receiver. The sensitiveness is in general limited by the difficulty of keeping a steady zero. By using a small transformer the sensibility can be much increased when the external circuit is of low resistance.

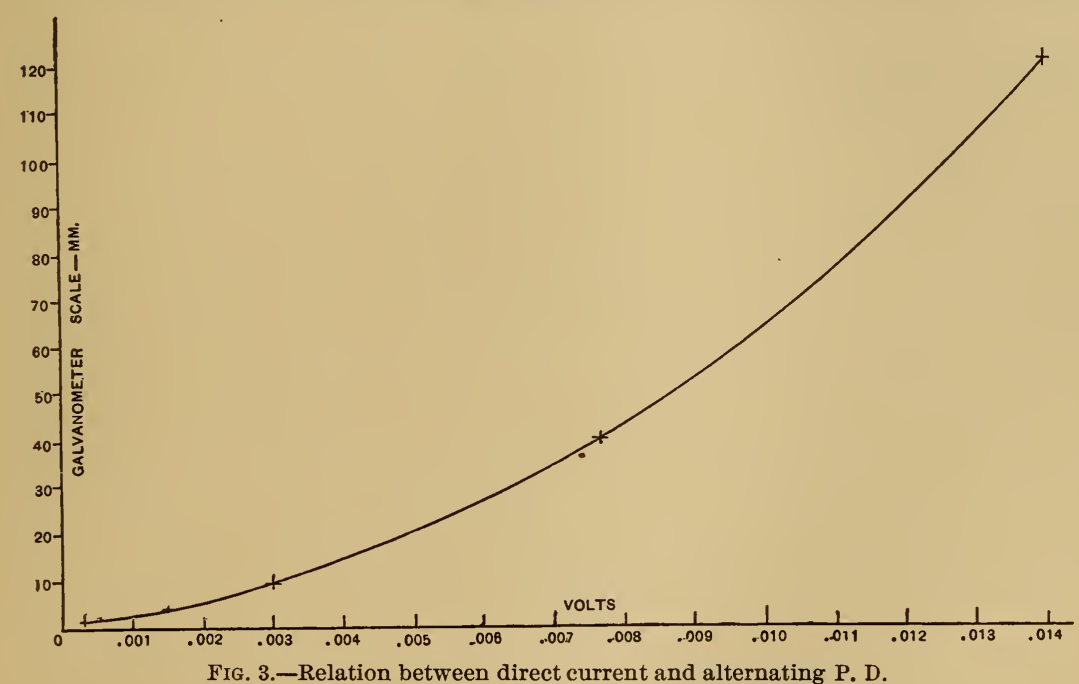

In this way I have sometimes obtained deflections of over $10 \mathrm{~cm}$ for 0.001 volt.

The action of the rectifier seems to be independent of frequency within the limits of ordinary laboratory practice. It responds equally well to a series of slow taps on the diaphragm of a telephone, or to a frequency of several thousand per second. It has also been found that it is sensitive to electrical waves, though less so and less regular in its action than the polarized platinum-point detector of Fessenden and Schlömilch. With a modified Blondlot apparatus it was found to be too sensitive to connect directly, but when connected to two small glass-tube condensers sliding on the wires of the secondary, as in the Rubens arrangement, the deflections increased or decreased, as the 
two circuits were thrown in or out of resonance. Waves could also be detected when the receiving apparatus was moved into the next room, and about 2 meters of wire were hung up in the doorway, as an aerial, 3 or 4 meters from the source of the waves. In these experiments with waves it is necessary that the small electrode be merely a point of wire, best sealed in glass, most of the experiments being made with No. 40 wire or smaller.

To secure the best results, the small electrode must be polished every two or three days, and the solution made up with pure $\mathrm{CuSO}_{4}$ and distilled water. The constancy of deflection is not by any means perfect, and unless it is found possible to keep the electrodes in a uniform condition, its main use must be as a zero instrument. I have also tested the same arrangement of a large and a small electrode of a number of other metals, not only in solutions of their own salts, but also in other electrolytes, but have found them less sensitive than copper in copper sulphate. 


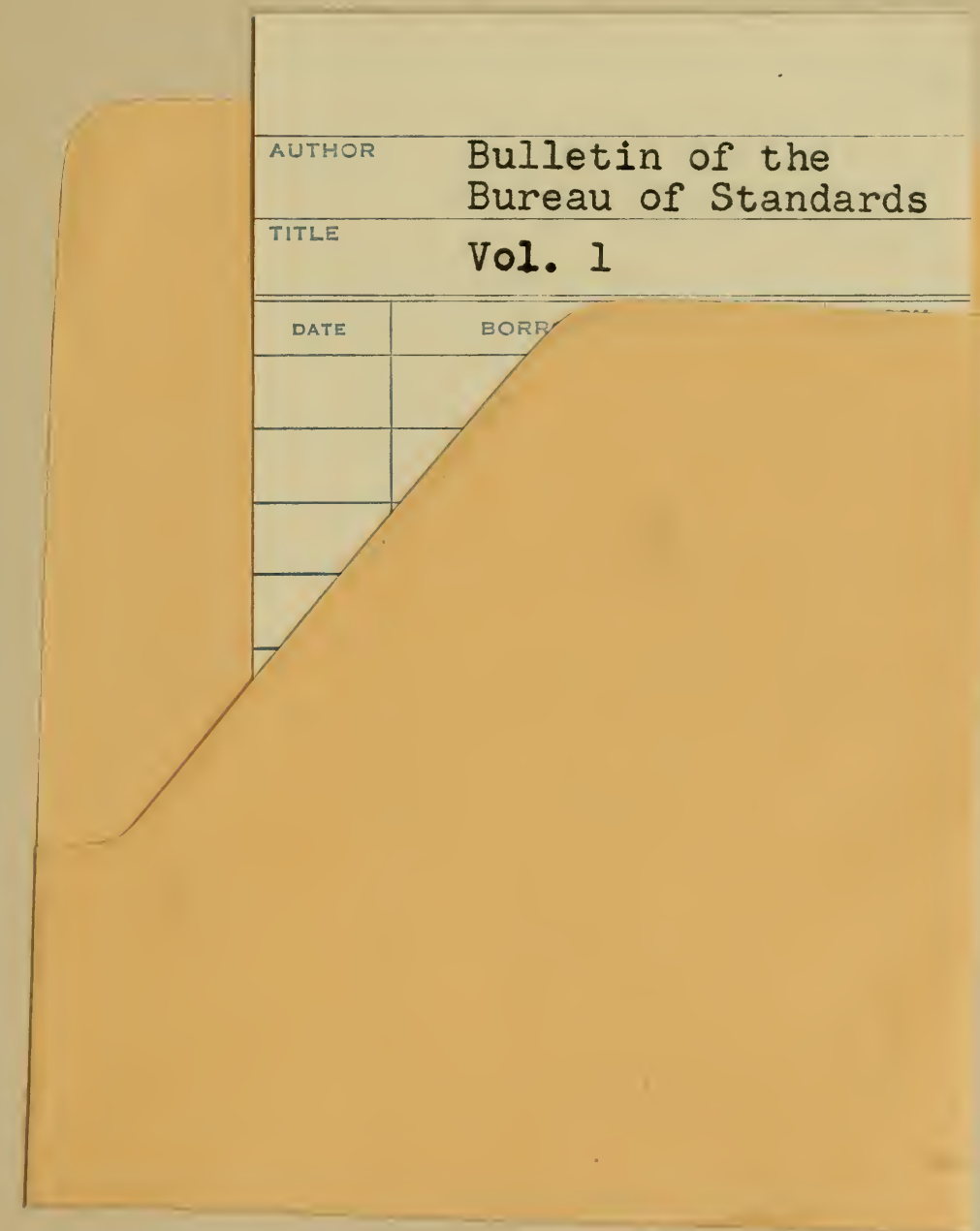


